\title{
Analysis of Acoustic Signals Interfering Diagnostic Measurements of Electric Power Transformer Tap Changers
}

\author{
T. Boczar*, A. Cichoń, S. Borucki And T. Szczyrba \\ Faculty of Electrical Engineering, Automatic Control and Computer Science, Opole University of Technology \\ Prószkowska 76, 45-758 Opole, Poland
}

\begin{abstract}
Subject matter of this paper applies to improvement of acoustic emission method used in the diagnosis of on load tap changers of high power transformers. During measurement of acoustic emission signals generated by damages or defects, which can appear in insulation system and in the power transformer tap changers, various forms of noise signals may occur. Interferences occurring during measurements under laboratory and under industrial conditions can be generated either by sources placed inside the studied electric power device or they can be emitted in their immediate vicinity. Sources of interfering signals are physical phenomena of a mechanical, electrical or acoustical nature. Interferences appearing during measurement of acoustic emission pulses generated by electrical discharges and other types of defects that overlap the measured waveforms may result in their distortion. As part of the research conducted, the results of which are presented in this paper, following was subjected under consideration: measurements of acoustic noise signals generated by partial surface discharges occurring on support and bushing insulators were performed.
\end{abstract}

DOI: $10.12693 /$ APhysPolA.124.387

PACS: 43.58.-e, 43.60.-c, 43.60.Bf

\section{Introduction}

During the measurements of acoustic emission (AE) pulses generated by partial discharges (PDs) in the insulation of power appliances various forms of interfering signals occur. The disturbances occurring during measurements in laboratory and industrial conditions can be generated by the sources inside the power appliances under study as well as the sources emitted in their surrounding. The sources of interfering signals can be physical phenomena of a mechanical, electric or acoustic character. The disturbances which occur during measurement of $\mathrm{AE}$ pulses generated by PDs when overlapping the time runs measured can cause their deformation. This phenomenon can have a direct influence on the correct interpretation of the measurement results obtained using the AE method. Hence the necessity of evaluation of the influence of interfering signals on the runs of $\mathrm{AE}$ pulses generated by PDs. Therefore the AE signals generated by disturbance sources should be measured and their frequency and time-frequency analysis carried out. The results obtained in this way can be the basis for a comparative analysis of AE signals in order to identify particular types of disturbances. Based on the results of the comparative analysis of acoustic interfering signals and $\mathrm{AE}$ pulses generated by PDs it is possible to select a filter eliminating adequate frequency ranges [1-5].

The subject matter of this paper refers to the analysis of the influence of the interfering acoustic signals generated by fans and pumps cooling transformer oil

*corresponding author; e-mail: t.boczar@gmail.com on the measurement results of PDs taken by using the AE method. The paper presents the results of measurements and frequency and time-frequency analyses using short-time Fourier transform (STFT) of the acoustic signals generated by the measured sources of disturbances. Moreover, the influence of the disturbances analyzed on the measurement results of the $\mathrm{AE}$ pulses generated by basic PD forms that can occur in paper-oil insulation systems was determined [6-8].

\section{Characteristics of the sources of interfering signals, the measuring apparatus used, and metrological conditions}

The measurements of interfering signals that occur during a regular operation of the triphase oil-immersed autotransformer type TLSRB 250000/400 PN were taken in industrial conditions in a power station. The autotransformer under study was of the following nominal data: active power $250 / 250 / 50 \mathrm{MW}$, prime voltage $400 \mathrm{kV}$, secondary side voltage $123 \pm 8 \times 1.5 \% \mathrm{kV}$, connection system Ynyn0/d11. Within the research measurements were taken of the AE signals generated by a cooling fan and of those induced by the operation of the pumps forcing the movement of insulation oil in a closed circulation.

A standard system by the firm Brüel\&Kjær, consisting of the following elements: a wide-band contact piezoelectric transducer, an amplifier equipped with a linear filter, and a measuring card, was used for the measurement of the AE signals. A detailed characteristics of the measuring apparatus used and a description of the computational procedure have been shown, among others, in the paper [7]. 


\section{Results of frequency analysis of the acoustic disturbance signals}

Figures 1-4 show power density spectra (PDS) (Figs. 1, 3) and pseudospectrum MUSIC of the acoustic interfering signals generated by the interference sources under study. The characteristics in Figs. 1 and 2 show the runs obtained for the interfering signals generated by a fan, and Figs. 3 and 4 by the pumps cooling insulation oil in the autotransformer under study.

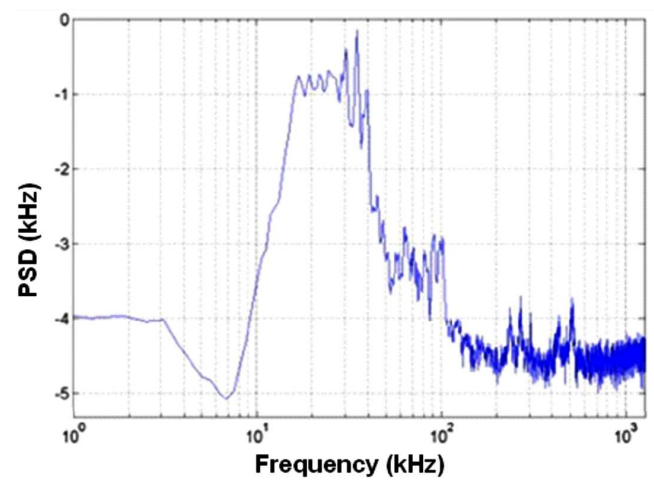

Fig. 1. PDS run obtained for the acoustic interfering signals generated by the cooler fans of the autotransformer.

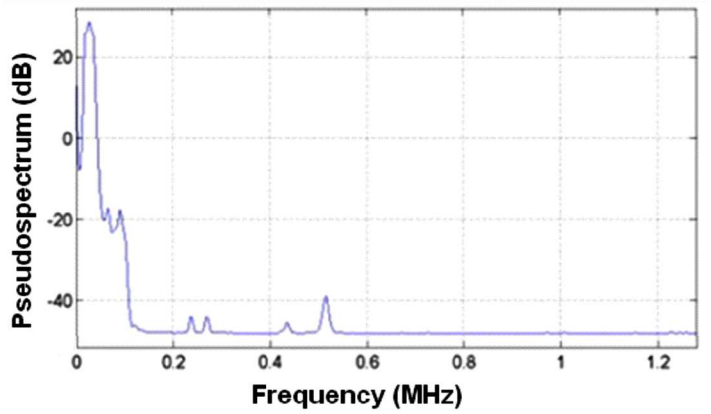

Fig. 2. Pseudospectrum MUSIC run obtained for the acoustic interfering signals generated by the cooler fans of the autotransformer.

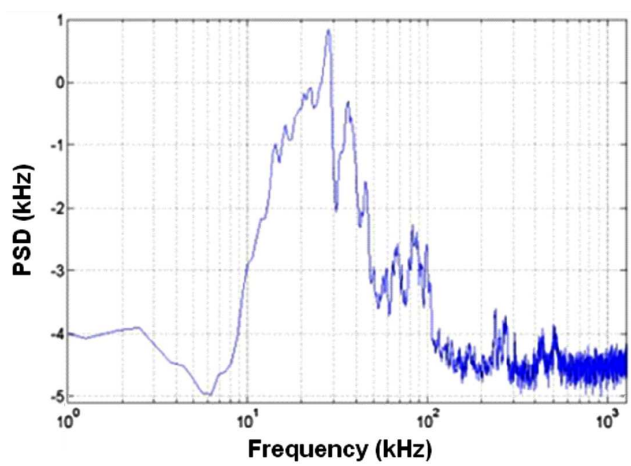

Fig. 3. PDS run obtained for the acoustic interfering signals generated by the cooling pumps of the autotransformer.

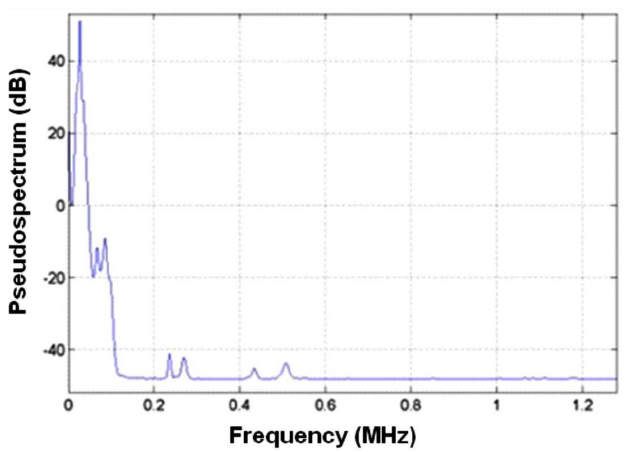

Fig. 4. Pseudospectrum MUSIC run obtained for the acoustic interfering signals generated by the cooling pumps of the autotransformer.

\section{Results of time-frequency analysis of the acoustic disturbance signals}

Figures 5-10 show spectrograms on which timefrequency images were drawn and for which a threshold function, cutting off the components of lower amplitude values, was used to set off the coherent structures obtained. Also three-dimensional spectrograms of the power spectrum density (Figs. 7,8 ) and the amplitude

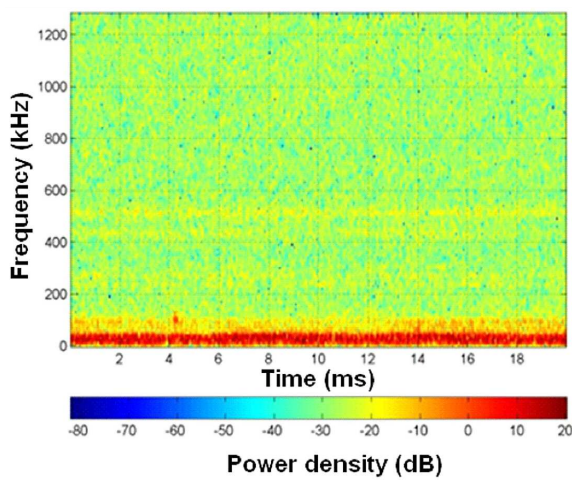

Fig. 5. Spectrogram calculated by using STFT for the acoustic interfering signals generated by the cooler fans of the autotransformer.

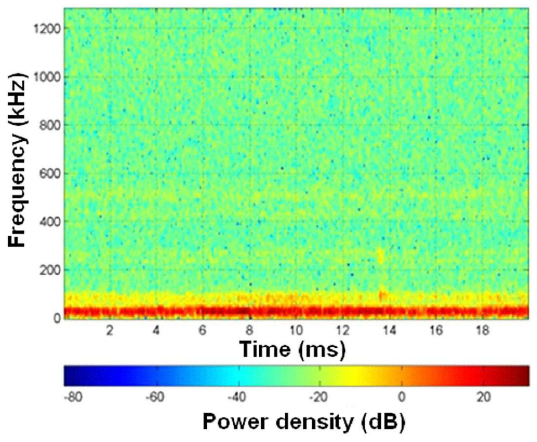

Fig. 6. Spectrogram calculated by using STFT for the acoustic interfering signals generated by the cooling pumps of the autotransformer. 


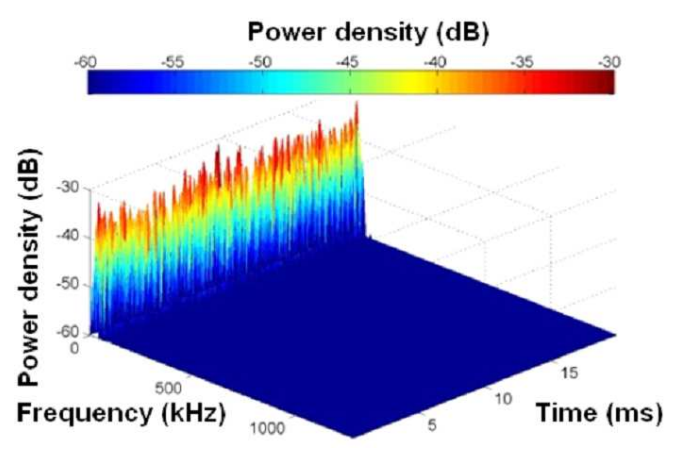

Fig. 7. Three-dimensional spectrogram of the power spectrum density obtained for the acoustic interfering signals generated by the cooler fans of the autotransformer.

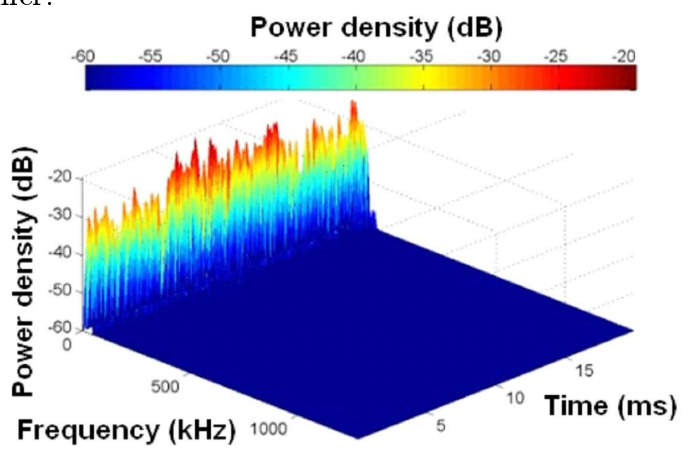

Fig. 8. Three-dimensional spectrogram of the power spectrum density obtained for the acoustic interfering signals generated by the cooling pumps of the autotransformer.

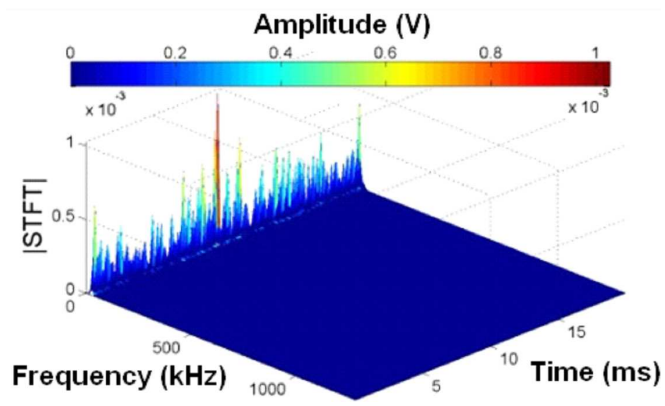

Fig. 9. Three-dimensional spectrogram of the amplitude obtained for the acoustic interfering signals generated by the cooler fans of the autotransformer.

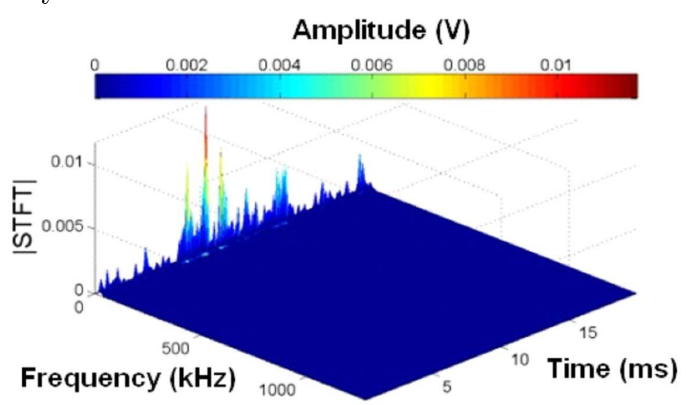

Fig. 10. Three-dimensional spectrogram of the amplitude obtained for the acoustic interfering signals generated by the cooling pumps of the autotransformer.
(Figs. 9, 10), which were determined for the AE pulses generated by the interfering signals under study, were shown for graphic presentation.

\section{Analysis of the results obtained}

Analyzing the results obtained the following conclusions can be drawn:

- The range of dominant frequencies of the frequency characteristics drawn for the acoustic interfering signals generated by the cooling fan of the autotransformer was within $0-70 \mathrm{kHz}$.

- For the frequency spectrum runs determined for the AE signals generated by the operation of the autotransformer pumps the range of dominant frequencies was within $30-60 \mathrm{kHz}$.

- Analyzing the results obtained during measurements of PDs using the AE method the influence of the interfering signals dominant bands of which overlap the spectra of the measured AE pulses generated by PDs should be taken into consideration. The resultant range of dominant frequencies for the interfering signals measured, for the discrimination threshold of $-20 \mathrm{~dB}$, should be within the range from 0 to $70 \mathrm{kHz}$.

\section{Comparative analysis of the acoustic emission pulses generated by partial discharges and interference sources under study}

Eight basic PD forms that can occur in insulation oil were selected for the comparative analysis with the interference sources under study. The research work carried out referred to PDs generated in the systems that enable modeling of the following discharge types: class 1 - discharges in the point-point system in oil, which can be related to PDs generated by insulation damage of two neighboring turns of winding of a transformer; class 2 discharges in the point-point system in oil with gas bubbles, which can reflect PDs in gassy oil and which are caused by insulation damage of two neighboring turns of winding of a transformer; class 3 - discharges in the point-plane system in oil, which can model PDs occurring between a damaged part of transformer winding insulation and earthed flat parts (tube elements); class 4 - discharges in the surface system of two flat electrodes with paper-oil insulation between them, the most common PD form occurring in the so-called triple point, in which an electrode surface is in contact with solid and liquid dielectrics; class 5 - discharges in the surface system of one flat electrode and one multipoint electrode, with paper-oil insulation between them, a different electric field intensity distribution in comparison with the surface system with two flat electrodes; class 6 - discharges in the multipoint-plane system in oil, which can model PDs occurring between a multipoint damage of transformer winding insulation and earthed flat parts (tube elements); class 7 - discharges in the multipointplane system in oil with gas bubbles, which can be connected with PDs occurring between a multipoint damage 
of transformer winding insulation and earthed flat parts (tube elements), but in oil with gas molecules; class 8 discharges on indeterminate potential particles moving in oil, which can model PDs occurring in oil containing particles of cellulose fibers formed in the process of a gradual degradation of paper-oil insulation caused by aging processes.

A detailed characteristics of the conditions in which the experiments were performed and the results of the frequency and time-frequency analyses carried out of the $\mathrm{AE}$ pulses generated by the PD forms have been presented, among others, in the papers $[4,6]$.

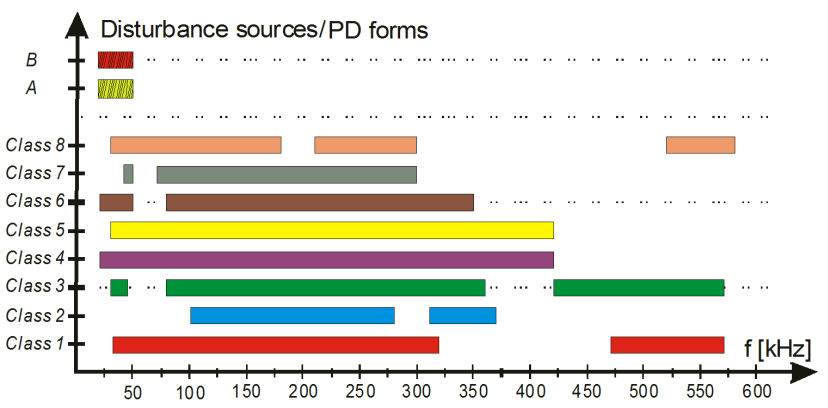

Fig. 11. Comparative listing of dominant frequency bands for the AE pulses from PDs in oil (class1-class8) and disturbance sources $(A, B)$.

A comparative listing of the results of the frequency and time-frequency analyses of the AE pulses generated by the PD forms under study and the acoustic interfering signals are shown in Fig. 11, on which the ranges of the dominant frequency bands have been shown in the form of columns. Letters were used to mark the interference sources under study, adopting the following way of numeration of the interfering acoustic signals: $A-$ generated by operating cooler fans autotransformer; $B$ - generated by pumps inducing autotransformer insulation oil circulation.

\section{Summary}

During the analysis of the results obtained at diagnostic measurements of insulation systems of power transformers taken by using the $\mathrm{AE}$ method, the influence of the interfering signals should be taken into account as their bands of dominant frequencies overlap the spectra of the measured AE pulses generated by PDs. The resultant interval of dominant frequencies for the interfering signals measured, for the discrimination threshold $-20 \mathrm{~dB}$, should be assumed in the range from 0 to $70 \mathrm{kHz}$. In order to eliminate the influence of the analyzed interference of the acoustic character, a high-pass filter of the low frequency equal to $70 \mathrm{kHz}$ or numerical procedures making a digital filtration of the AE signals registered possible can be used.

\section{Acknowledgments}

The work was financed with the means from the National Research and Development Center within LIDER program.

\section{References}

[1] E. Carminati, C. Loredana, L. Massimo, M. Antonello, IEEE Trans. Instrum. Measur. 50, 1413 (2000).

[2] M. Zdanowski, S. Wolny, D. Zmarzły, T. Boczar, J. Electrostat. 65, 239 (2007).

[3] L. Fuqi, J. Lei, Adv. Technol. Electr. Eng. 19, 69 (2000).

[4] D. Wotzka, T. Boczar, D. Zmarzły, Acta Phys. Pol. A 116, 428 (2009).

[5] K. Barczak, Bull. Pol. Acad. Sci., Techn. Sci. 59, 409 (2011).

[6] D. Wotzka, D. Zmarzły, T. Boczar, Acta Phys. Pol. A 118, 1272 (2010).

[7] S. Borucki, T. Boczar, A. Cichoń, Arch. Acoust. 36, 49 (2011).

[8] A. Kyprianou, P.L. Lewin, V. Efthimiou, A. Stavrou, G.E. Georghiou, Measur. Sci. Technol. 17, 2367 (2006). 